\title{
Time spectra of a nearly-single-line pure nuclear reflection excited by synchrotron radiation
}

\author{
A. I. Chumakov, M. V. Zelepukhin, and G. V. Smirnov \\ I. V. Kurchatov Institute of Atomic Energy, 123182 Moscow, U.S.S.R. \\ U. van Bürck \\ Physik-Department (E15), Technische Universität München, D-8046 Garching bei München, Germany \\ R. Rüffer, R. Hollatz, H. D. Rüter, and E. Gerdau \\ II. Institut für Experimentalphysik, Universität Hamburg, D-2000 Hamburg 50, Germany
}

(Received 13 February 1990)

\begin{abstract}
The time dependence of the coherent response of a ${ }^{57} \mathrm{FeBO}_{3}$ single crystal excited by synchrotron-radiation pulses was studied in the pure nuclear (333) Bragg reflection near the Néel temperature, where the Mössbauer reflection spectrum consists of nearly a single line of almost natural linewidth. The time spectra revealed a delayed reemission of $\gamma$ quanta into the Bragg direction starting at zero intensity. This behavior was determined by two factors: the coherent decay of the nuclear excited state and the interference of two nearly coinciding hyperfine components.
\end{abstract}

\section{INTRODUCTION}

Mössbauer radiation sources of very high brilliance will be available in the near future at dedicated synchrotronradiation (SR) facilities. For Mössbauer spectroscopy, narrow single-line sources are essential in the case of time-integral measurements. Therefore possibilities of Mössbauer single-line filtering of synchrotron radiation are searched for.

The most advanced method of Mössbauer filtering is based on pure nuclear diffraction by single crystals. However, pure nuclear diffraction is usually intimately related to the hyperfine interaction of nuclear magnetic or quadrupole moments with the internal crystalline fields. ${ }^{1-3}$ Therefore the energy spectrum of the filtered radiation contains several hyperfine components. In the time spectrum of the filtered radiation, the interference of these components gives rise to quantum beats. ${ }^{4-6}$

One possibility to obtain a single-line source will be the elimination of all hyperfine components but one by an additional resonance absorber. ${ }^{7}$ Another way used in the present work is based on the possibility of directly obtaining a single-line pure nuclear reflection in $\mathrm{FeBO}_{3}$ heated up to the Néel temperature where the magnetic hyperfine interaction is vanishing. ${ }^{8}$ The first method yields a single line of several natural linewidths, since in nuclear Bragg diffraction the reflection lines are usually considerably broadened due to dynamical diffraction effects. The second method yields a single line of almost natural linewidth because it is the superposition of several lines which add destructively at their wings.

The aim of the present work was the study of the time dependence of the coherent nuclear response of a ${ }^{57} \mathrm{FeBO}_{3}$ single crystal excited by SR under the condition of vanishing magnetic hyperfine splitting at the Néel temperature.

\section{EXPERIMENT AND RESULTS}

The time dependence of the coherent resonant $\gamma$ reemission was measured at the Mössbauer station of the HASYLAB [Hamburger Synchrotronstrahlungslabor, Deutsches Elektronen-Synchrotron (DESY)]. ${ }^{9}$ The pure nuclear reflection ${ }^{57} \mathrm{FeBO}_{3}$ (333) was employed. The crystal was mounted in a special diffraction cell as in a previous experiment, where the temperature dependence of the reflection spectra in-between room and Néel temperature was studied. ${ }^{10}$ The crystal was heated up to temperatures slightly above the Néel temperature $\left(T_{N}\right.$ $=75.2{ }^{\circ} \mathrm{C}$ ). A weak magnetic field of about $10 \mathrm{mT}$ was applied to the crystal perpendicular to the scattering plane in order to induce antiferromagnetic order above the Néel temperature. ${ }^{8}$ This weak external field served to expand the critical temperature range where a nearly-single-line Mössbauer spectrum can be observed. The time spectra were measured at three different temperatures between 75.5 and $75.8^{\circ} \mathrm{C}$. For comparison, the Mössbauer spectra of this reflection were measured at about the same temperatures using an intense ${ }^{57} \mathrm{Co}$ source at the Kurchatov Institute. The results of both measurements are shown in Fig. 1.

The energy spectra consist of a single line, which is slightly asymmetric at the base. The reflection intensity decreased with increasing temperature. The intensities measured at the three temperatures were about $20 \%, 10 \%$, and $5 \%$ relative to the intensity of the full spectrum measured at room temperature, respectively (or $50 \%, 25 \%$, and $12 \%$ relative to the intensity of an outer line). The energy width of the reflection is $\sim 3.1$ natural linewidths, where the source linewidth of 2.1 natural linewidths has to be subtracted from this measured linewidth.

The time spectra of the reflection reveal that the pulsed 

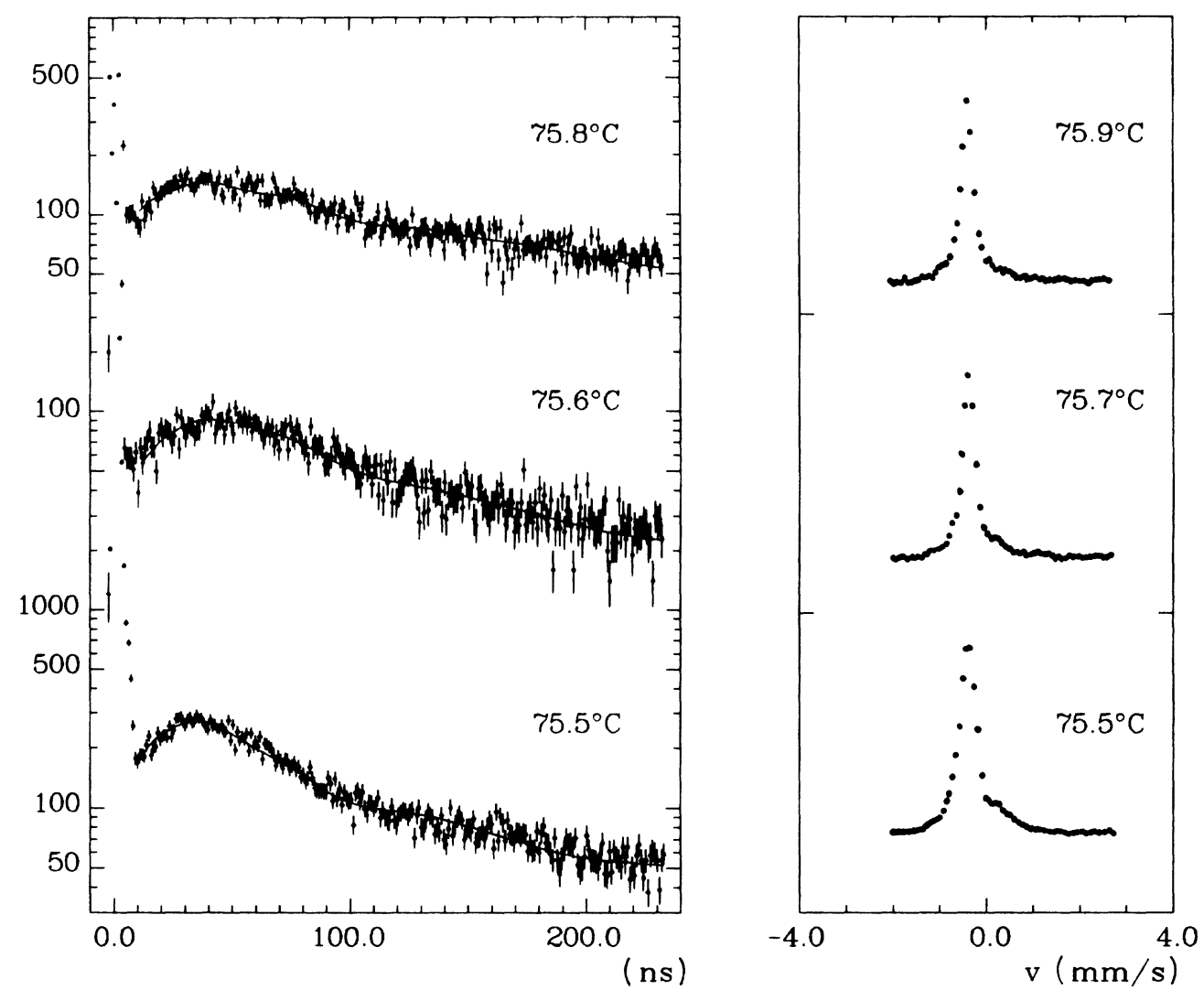

FIG. 1. Time spectra of the nuclear response to the pulsed excitation by synchrotron radiation in the pure nuclear reflection ${ }^{57} \mathrm{FeBO}_{3}$ (333) and the corresponding Mössbauer diffraction spectra, measured at temperatures slightly above the Néel point. The solid lines are fits to the spectra with magnetic fields of $1.5(0.2) \mathrm{T}, 1.3(0.2) \mathrm{T}$, and $0.5(0.2) \mathrm{T}$, respectively.

excitation of the nuclear system is followed by a delayed reemission into the Bragg direction. The reflection starts at zero intensity and increases until a maximum is reached at about 30-50 ns. Afterwards it decreases slowly. The solid curves in the time spectra in Fig. 1 are the results of a fit with the full dynamical theory. The quadrupole splitting $\Delta=\frac{1}{2} e Q V_{z z}$ was $-0.35 \mathrm{~mm} / \mathrm{s}$ for all spectra, so only the magnetic field, the background, and a normalization factor were fitted. The $\chi^{2}$ values were all below 1.1 .

\section{INTERPRETATION AND DISCUSSION}

The energy spectra of the reflection demonstrate that in stationary scattering conditions a nearly single line of Mössbauer radiation of almost natural linewidth can be filtered from synchrotron radiation. This situation is achieved in antiferromagnetic $\mathrm{FeBO}_{3}$ when near the Néel point the magnetic hyperfine interaction breaks down and becomes weaker than the electric interaction, which has an axis perpendicular to the magnetization axis. The Mössbauer absorption spectrum then collapses to a quadrupole doublet where each line is further broadened by the magnetic field. The diffraction spectrum, by contrast, is dominated by only one of the doublet lines. This can be understood from the splitting of the excited $\frac{3}{2}$ level by the combined interaction. The electric interaction alone creates two sublevels. The further splitting of these sub- levels by the magnetic interaction is very different. One level splits linearly with the magnetic field, whereas the other splits only in higher order. ${ }^{11}$ Two groups of transitions to the only magnetically split ground state occur. They built a quadrupole doublet. Since in the present case of a pure nuclear reflection of magnetic origin the reflection intensity increases strongly with the magnetic line splitting, only one line appears in the diffraction spectrum.

The time spectra, i.e., the time evolution of the radiative emission into the Bragg direction, can be explained by a process determined by two factors: the decay of the nuclear excited state and the interference between the nuclear hyperfine components, which results in the quantum beats. ${ }^{12}$ The time evolution of the resonance fluorescence strongly depends on the ratio between two characteristic times: the decay time $\tau$ and the beat period $T$.

If $T \ll \tau$, the interference between the different hyperfine components gives rise to a beating of the reemitted intensity, which, on the average, follows the decay of the excited state. Changes from constructive to destructive interference and vice versa occur so many times during the lifetime of the excited state that the decay of the nuclear excitation looks nearly the same in any allowed coherent emission channel. If, however, $T \geq \tau$ or even $T \gg \tau$, the situation is completely changed. In the limit $T \gg \tau$, the interference in the Bragg direction is destructive during the lifetime. The conditions of the present ex- 
periment were $T \geq \tau$, since the hyperfine splitting was in the order of the linewidth. In this intermediate situation decay and interference compete: the transition from constructive to destructive interference (or vice versa) in the $\gamma$-ray emission occurs just in times comparable to the lifetime. For the electronically forbidden (333) reflection the initial interference is destructive. The transition to constructive interference proceeds so slowly that most of the stored excitation energy is already released. This explains why only a delayed and relatively low intensity was observed in the Bragg direction.

The delayed reemission into the Bragg direction could give the impression that the decay was delayed altogether. This behavior would then be in strong contrast to the speedup of the decay observed at room temperature. ${ }^{5}$ For the characterization of the decay of the nuclear excitation, however, one has to take into account all possible channels of reemission. After the prompt interruption of the excitation, the reemission is mainly influenced by the coherent channels. In these nonstationary conditions two coherent channels are allowed, the Bragg and the forward channels. Since the forward reemission is initially constructive, a strong decay channel is opened immediately after the excitation by the synchrotron flash. A strong speedup of the decay occurs. Under the conditions of slow beating, the forward channel stays open long enough so that the speeded-up decay carries away most of the excitation energy into the forward direction. In the Bragg reflection direction the decay is not observed until very late times, when the strong initial speedup is already over.
It is interesting to note that the time spectra give important information about the physical nature of the energy spectra. The nearly single line observed in the energy spectra could be of different origin, being a true single line or the sum of two single lines scattering either in phase or in antiphase. A true single line or the sum of two single lines scattering in phase would yield a time dependence with initial maximum intensity. This is in contrast with the observed time spectra, where the initial intensity is zero corresponding to the antiphase scattering of two superimposed single lines. Thus the present case of antiferromagnetic single-line filtering can be distinguished from the (so far) not yet observed case of single-line filtering (e.g., by an electronically allowed reflection) by just the start of the time spectrum.

In this experiment the possibility of narrow single-line Mössbauer filtering of synchrotron radiation has been demonstrated. The pure nuclear reflection ${ }^{57} \mathrm{FeBO}_{3}$ (333) yields at the Néel temperature a beam of nearly-singleline Mössbauer radiation of almost natural linewidth, which can be employed in Mössbauer spectroscopy for time-integral measurements.

\section{ACKNOWLEDGMENTS}

This work has been funded by the Bundesministerium für Forschung und Technologie (Bonn, Germany) under Contracts No. 05270-GUI9 and No. KA1TUM 4/7 and by the I. V. Kurchatov Institute.
${ }^{1}$ G. V. Smirnov, V. V. Sklyarevskii, R. A. Voskanyan, and A. N. Artemev, Pis'ma Zh. Eksp. Teor. Fiz. 9, 123 (1969) [JETP Lett. 9, 70 (1969)].

${ }^{2}$ R. M. Mirzababaev, G. V. Smirnov, V. V. Sklyarevskii, A. N. Artemev, A. N. Israilenko, and A. V. Babkov, Phys. Lett. 37A, 441 (1971).

${ }^{3}$ E. Gerdau, R. Rüffer, H. Winkler, W. Tolksdorf, C. P. Klages, and J. P. Hannon, Phys. Rev. Lett. 54, 835 (1985).

${ }^{4}$ E. Gerdau, R. Rüffer, R. Hollatz, and J. P. Hannon, Phys. Rev. Lett. 57, 1141 (1986).

${ }^{5}$ U. van Bürck, R. L. Mössbauer, E. Gerdau, R. Rüffer, R. Hollatz, G. V. Smirnov, and J. P. Hannon, Phys. Rev. Lett. 59, 355 (1987).

${ }^{6}$ G. Faigel, D. P. Siddons, J. B. Hastings, P. E. Haustein, J. R. Grover, and L. E. Berman, Phys. Rev. Lett. 61, 2794 (1988).

${ }^{7}$ U. van Bürck, R. L. Mössbauer, E. Gerdau, W. Sturhahn, H. D. Rüter, R. Rüffer, A.I. Chumakov, M. V. Zelepukhin, and
G. V. Smirnov (unpublished).

${ }^{8}$ G. V. Smirnov, M. V. Zelepukhin, and U. van Bürck, Pis'ma Zh. Eksp. Teor. Fiz. 43, 274 (1986) [JETP Lett. 43, 352 (1986)].

${ }^{9}$ R. Rüffer, D. Giesenberg, H. D. Rüter, R. Hollatz, E. Gerdau, J. Metge, K. Ruth, W. Sturhahn, M. Grote, and R. Röhlsberger, in Proceedings of the International Conference on the Applications of the Mössbauer Effect, Budapest, 1989 [Hyperfine Interact. (to be published)].

${ }^{10}$ H. D. Rüter, R. Rüffer, E. Gerdau, R. Hollatz, A. I. Chumakov, M. V. Zelepukhin, G. V. Smirnov, and U. van Bürck, in Proceedings of the International Conference on the Applications of the Mössbauer Effect (Ref. 9).

${ }^{11}$ E. Matthias, W. Schneider, and R. M. Steffen, Ark. Fys. 24, 97 (1963).

12 J. P. Hannon and G. T. Trammell, Physica B 159, 161 (1989). 